\title{
FRECUENCIA DE LIPIDOSIS HEPÁTICA ENTRUCHAARCOÍRIS (Oncorhynchus mykiss) DE FASE JUVENIL EN UNA PISCIGRANJA DE LA SIERRA CENTRAL DEL PERÚ
}

\author{
Frequency of Hepatic Lipidosis in Juvenile Rainbow Trout (ONCorhynchus \\ mykiss) in a Fish Farm in the Peruvian Central Sierra
}

\section{Sandra Rosenthal N. ${ }^{1}$, Nieves Sandoval C., ${ }^{1,3}$, César Gavidia C. ${ }^{2}$, Luis Tabacchi N. $^{1}$}

\section{Resumen}

El objetivo del estudio fue determinar la frecuencia de lipidosis hepática en la trucha arcoíris (Oncorhynchus mykiss) de fase juvenil en base a lesiones anátomohistopatológicas en el hígado. Se trabajó con 58 peces de una piscigranja comercial de la sierra central del Perú. Los peces fueron clasificados como sanos $(n=23)$ y enfermos $(n=35)$ según el oscurecimiento de la piel y erosión de las aletas. Se colectaron muestras de hígado, que se fijaron en formol al $10 \%$ y fueron coloreadas con Hematoxilina-eosina. El 70.6\% (41/58) de los peces presentó lesiones compatibles con lipidosis hepática, siendo el grado 3 el de mayor presentación $(27.6 \%)$. Las mayores frecuencias de lipidosis hepática en grados 3 y 4 se observaron en animales sanos.

Palabra clave: lipidosis, hígado, frecuencia, trucha arcoíris

\section{Abstract}

The objective of the study was to determine the frequency of hepatic lipidosis in rainbow trout (Oncorhynchus mykiss) of juvenile phase based on anatomical and histopathological lesions in the liver. The specimens $(n=58)$ were obtained from a commercial fish farm in the central highlands of Peru. The fish were classified as healthy $(n=23)$ and ill $(n=35)$ according to the darkening of the skin and fin erosion. Liver samples were collected, fixed in $10 \%$ formalin, and stained with hematoxylin eosin. Results showed that $70.6 \%$ (41/58) of the fish had lesions compatible with hepatic lipidosis, where grade 3 had the largest incidence $(27.6 \%)$. The highest frequency of hepatic lipidosis in grade 3 and 4 was observed in healthy animals.

Key words: lipidosis, liver, frequency, rainbow trout

\footnotetext{
${ }^{1}$ Laboratorio de Histología, Embriología y Patología, ${ }^{2}$ Laboratorio de Medicina Veterinaria Preventiva, Facultad de Medicina Veterinaria, Universidad Nacional Mayor de San Marcos, Lima

${ }^{3}$ E-mail: nieves.sandovalchaupe@gmail.com
} 


\section{INTRODUCCIÓN}

La acuicultura es una de las actividades emergentes en el Perú; no obstante, el cultivo de trucha arcoíris (Oncorhynchus mykiss) es una actividad que se viene realizando desde hace muchos años, y que ha ubicado al Perú como el tercer exportador mundial de truchas (Cowey y Roberts, 1983; Blanco, 1995).

La lipidosis hepática es la excesiva acumulación de grasa, principalmente de grasa neutra (triglicérido) en el citoplasma del hepatocito (Hibiya, 1982; Carlyle y Duncan, 1990; Trigo, 1998). Tiene consecuencias en el rendimiento productivo ocasionando una disminución en el crecimiento (Gang et al., 2006) y deficiente conversión alimenticia; sin embargo no ocasiona altas tasas de mortalidad en la trucha (Cowey y Roberts, 1983).

Todos los salmónidos son susceptibles a la lipidosis hepática, siendo un problema particularmente importante en la trucha arcoíris (Roberts y Shepherd, 1980; Roberts, 1983). Se ha reportado la presencia de la enfermedad en el salmón del Atlántico (Salmo salar) (Roald, 1976; Ferguson, 2006), la trucha del arroyo (Salvelinus fontinalis), el pez gato americano (Ietalurus punctatus), carpa común (Cyprinus carpio) (Erdal y Ercument, 2006) y, en menor frecuencia, en la trucha café (Salmo trutta) (Ghittino y León, 1963; Ferguson, 1989). Estudios realizados en Chile mostraron una frecuencia de lipidosis hepática en salmónidos de crianza intensiva de 46\% (López et al., 2001) y de 51\% (182/355) (Cubillos et al., 2001).

En la piscicultura nacional, el conocimiento de los problemas sanitarios relacionados a la alimentación es escaso e incompleto. No se dispone de estudios de frecuencia de lipidosis hepática en truchas arcoíris ni su repercusión en la producción de truchas de cultivo; sin embargo, hay reportes de observaciones, tanto macroscópicas como microscópicas en hígados que señalan la presencia del proceso degenerativo (N. Sandoval, Lima, comunicación personal). El presente estudio se hizo para determinar la frecuencia de lipidosis hepática en la trucha arcoíris de fase juvenil en la Sierra Central del Perú.

\section{Materiales y Métodos}

\section{Lugar de Estudio y Animales}

El estudio se llevó a cabo en la Piscigranja Pachacayo, de la SAIS (Sociedad Agraria de Interés Social) Túpac Amaru, ubicada a $3600 \mathrm{msnm}$, en el distrito de Chanchayllo, provincia de Jauja, departamento de Junín, durante los meses de junio a julio de 2006.

Se emplearon 58 truchas de fase juvenil (7-8 meses de edad), con un peso promedio de $125 \mathrm{~g}$. El tamaño de la muestra estuvo de acuerdo al Teorema del Límite Central (Spiegel, 1991). Los peces fueron seleccionados al azar en los estanques de juveniles.

\section{Toma de Muestras y Análisis Histológico}

Los animales fueron sacrificados mediante la técnica del corte medular. Se observó la condición física, superficie del cuerpo y erosión de aletas. La necropsia se llevó a cabo siguiendo la metodología descrita por Ostrander (2000). Se realizó el examen macroscópico del hígado, tomándose segmentos de 3 a $4 \mathrm{~mm}$, que fueron fijados en formalina tamponada al $10 \%$ y teñidos con Hematoxilina-Eosina.

Se realizó una tinción Gram de las láminas para determinar la presencia de bacterias dentro de los cortes de hígado, además de la identificación de granulomas y del hongo Ictiofonus spp dentro del parénquima hepático.

El análisis histológico de los grados de intensidad para lipidosis hepática se basó en la clasificación descrita por Bernet et al. 
Cuadro 1. Grado de severidad y extensión de lesiones en hígado causada por lipidosis hepática a nivel microscópico

\begin{tabular}{cll}
\hline Grado & Severidad & Extensión \\
\hline \multirow{2}{*}{0} & $\begin{array}{l}\text { Ausencia de vacuolas (imágenes negativas de la } \\
\text { grasa) de bordes definidos en el citoplasma. }\end{array}$ & Ninguna \\
& $\begin{array}{l}\text { Escasa presencia de microvacuolas, predominan- } \\
\text { temente de borde definido (se mantiene la arquitec- }\end{array}$ & $\begin{array}{l}\text { Multifocal, hasta } \\
25 \%{ }^{1} \text { de degene- } \\
\text { ración grasa }\end{array}$ \\
& $\begin{array}{l}\text { tura del hepatocito). } \\
\text { Leve presencia de micro o macrovacuolas de borde } \\
\text { definido, con mayor compromiso de las células }\end{array}$ & $\begin{array}{l}\text { Multifocal, con 25 a } \\
\text { he\% de degeneración } \\
\text { grasa }\end{array}$ \\
& $\begin{array}{l}\text { Moderadicas. } \\
\text { borde definido y núcleo ligeramente desplazado. }\end{array}$ & $\begin{array}{l}\text { Difuso, con 50 a 75\% } \\
\text { de degeneración grasa }\end{array}$ \\
& $\begin{array}{l}\text { Ligera distorsión de la arquitectura del hepatocito. } \\
\text { Severa presencia de macrovacuolas dentro del } \\
\text { citoplasma del hepatocito, núcleo aplanado y ovalado } \\
\text { ligeramente. Pérdida de la citoarquitectura de los } \\
\text { hepatocitos y muy unidos entre sí. }\end{array}$ & $\begin{array}{l}\text { Difuso, con 75 a } \\
100 \% \text { de degenera- } \\
\text { ción grasa }\end{array}$ \\
\hline
\end{tabular}

${ }^{1}$ El porcentaje se refiere a la sección de corte del hígado

(1999), y modificado por el laboratorio, añadiendo los porcentajes de degeneración grasa, según lo señalado en el Cuadro 1.

\section{Análisis de Datos}

Los resultados de frecuencia de las lesiones macroscópicas y trastornos microscópicos hepáticos se expresaron en forma porcentual.

\section{Resultados}

En el examen externo se pudo diferenciar la presencia de 23 animales aparentemente normales (sin lesiones aparentes) y 35 con lesiones externas, caracterizadas por oscurecimiento de la piel (melanosis) parcial y total, así como erosión de las aletas.

En la necropsia, la coloración pálida fue la lesión macroscópica de mayor frecuencia de los peces sanos $(34.8 \%, 8 / 23)$ y peces con lesiones o enfermos $(45.7 \%, 16 / 35)$. Los peces, identificados como aparentemente sanos durante el examen físico externo, evidenciaron, además, consistencia friable $(4.3 \%$, $1 / 23)$ y hemorragia difusa $(21.7 \%, 5 / 23)$ en el hígado (Cuadro 2).

El análisis histológico determinó que la frecuencia de lipidosis hepática fue de $70.6 \%$ (41/58), siendo el grado 3 el de mayor presentación (27.6\%). Las mayores frecuencias de lipidosis hepática en grados 3 y 4 se observaron en los animales sanos (Cuadro 3). Lesiones histológicas se muestran en la Fig. 1.

La lipidosis hepática se presentó, asimismo, en forma conjunta con trastornos de tipo infeccioso $(58.6 \%$; 34/58) asociados a ictiofonosis y con afecciones de tipo inflamatorio $(46.5 \%$; $27 / 58)$, que mayormente guardan relación con agentes infecciosos de tipo bacteriano. 
Cuadro 2. Frecuencia de lesiones macroscópicas en el hígado de trucha arcoíris (Oncorhynchus mykiss) de una piscigranja de Jauja, Junín (2006)

\begin{tabular}{lcccccc}
\hline \multirow{2}{*}{ Lesiones macroscópicas } & \multicolumn{2}{c}{ Sanos } & \multicolumn{2}{c}{ Enfermos $^{1}$} & \multicolumn{2}{c}{ Total } \\
\cline { 2 - 7 } & $\mathrm{N}^{\circ}$ & $\%$ & $\mathrm{~N}^{\circ}{ }^{\circ}$ & $\%$ & N. $^{\circ}$ & $\%$ \\
\hline Coloración pálida & 8 & 34.8 & 16 & 45.7 & 24 & 41.3 \\
Consistencia friable & 1 & 4.3 & 4 & 11.4 & 5 & 8.7 \\
Coloración amarilla & 0 & 0 & 3 & 8.6 & 3 & 5.1 \\
Hemorragia equimótica & 0 & 0 & 5 & 14.3 & 5 & 8.7 \\
Hemorragia difusa & 5 & 21.7 & 3 & 8.6 & 8 & 13.8 \\
Sin lesiones & 9 & 39.2 & 4 & 11.4 & 13 & 22.4 \\
\hline Total & 23 & 100.0 & 35 & 100.0 & 58 & 100.0 \\
\hline
\end{tabular}

${ }^{1}$ Peces con lesiones externas: melanosis parcial y total, erosión de las aletas

Cuadro 3. Frecuencia de lipidosis hepática en truchas arcoíris (Oncorhynchus mykiss), según el grado de lesión y el estado de salud (Jauja - Junín, 2006)

\begin{tabular}{|c|c|c|c|c|c|c|}
\hline \multirow{2}{*}{$\begin{array}{l}\text { Grado de lesión } \\
\text { hepática }^{2}\end{array}$} & \multicolumn{2}{|c|}{ Sanos } & \multicolumn{2}{|c|}{ Enfermo s $^{1}$} & \multicolumn{2}{|c|}{ Total } \\
\hline & N. ${ }^{\circ}$ & $\%$ & N. ${ }^{\circ}$ & $\%$ & N. ${ }^{\circ}$ & $\%$ \\
\hline 0 & 2 & 8.7 & 15 & 42.8 & 17 & 29.3 \\
\hline 1 & 3 & 13.0 & 5 & 14.2 & 8 & 13.8 \\
\hline 2 & 4 & 17.3 & 4 & 11.4 & 8 & 13.8 \\
\hline 3 & 7 & 30.4 & 9 & 25.8 & 16 & 27.6 \\
\hline 4 & 7 & 30.4 & 2 & 5.7 & 9 & 15.5 \\
\hline Total & 23 & 100 & 35 & 100 & 58 & 100 \\
\hline
\end{tabular}

${ }^{1}$ Peces con lesiones externas: melanosis parcial y total, erosión de las aletas

2 Según Bernet et al. (1999). Ver Cuadro 1

\section{Discusión}

La lesión macroscópica predominante fue la coloración pálida del hígado, tanto en peces sanos $(34.8 \%)$ como en aquellos con lesiones externas $(45.7 \%)$, siendo esta característica muy asociada a lipidosis hepática en campo, al igual que la consistencia friable.
Estas lesiones han sido reportadas también, en otras especies como el salmón del Atlántico (Salmo salar) (Roald y Armstrong, 1981).

La frecuencia de lipidosis hepática encontrada $(70.6 \%)$ fue superior a otros estudios realizados en Chile (Cubillos et al., 2001; López et al., 2001), donde se reporta frecuencias de $51.2 \%$ (182/355) y $45.6 \%$ (323/709), 


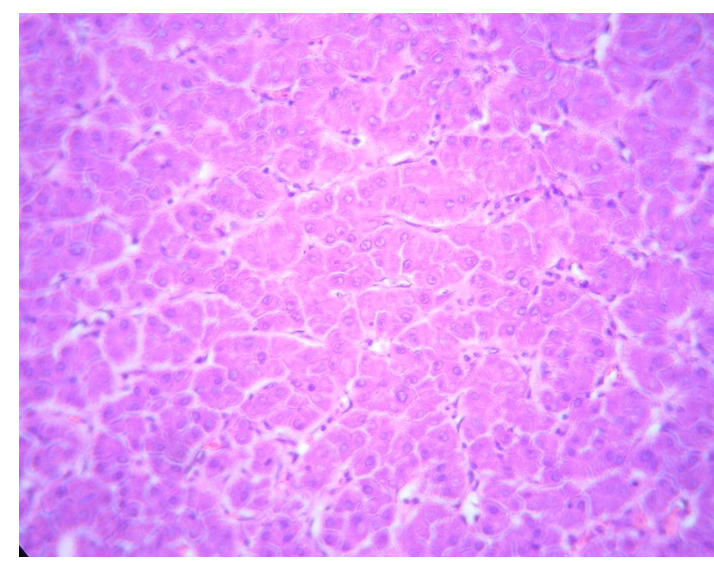

(a)

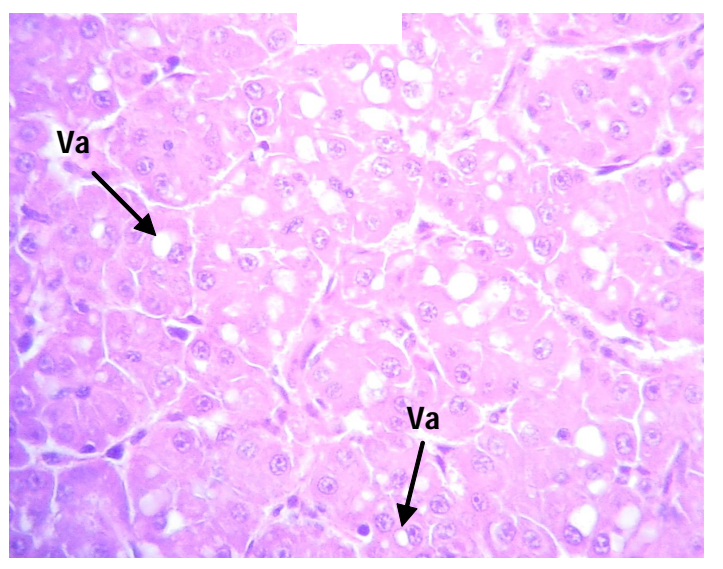

(c)

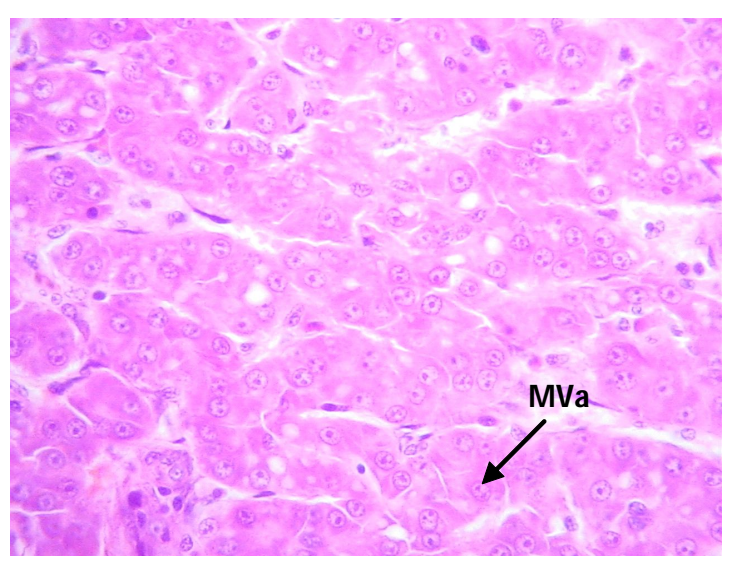

(b)

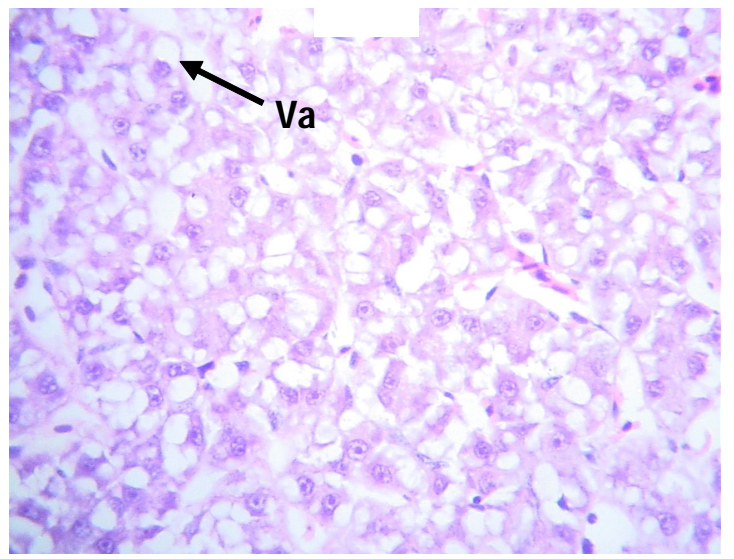

(d)

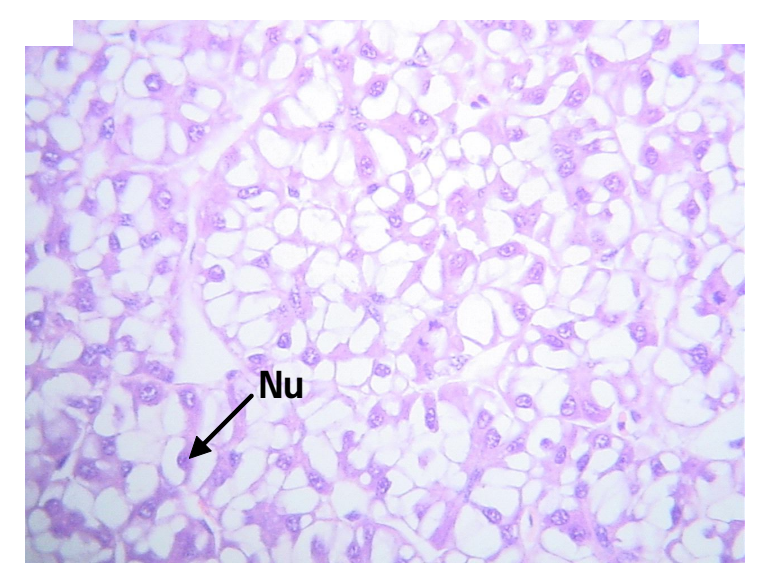

(e)

Figura 1. Cortes histológicos de hígado de trucha arcoíris (Oncorhynchus mykiss). (a) Tejido hepático normal, grado 0. (b) Grado 1. Microvacuola de borde definido (Mva). (c) Grado 2. Micro y macrovacuolas de borde definido (Va). (d) Grado 3. Micro y macrovacuolas de borde definido (Va), con mayor compromiso de tejido hepático. (e) Grado 4. Hepatocitos presentan un núcleo en la periferia y ligeramente ovalado $(\mathrm{Nu})$. HE, 400x 
respectivamente. Estas diferencias se deben posiblemente a la edad y especies evaluadas, tamaño muestral, época del año, así como a los criterios de inclusión de los peces en los estudios. No obstante, este elevado porcentaje de lipidosis hepática podría deberse a problemas metabólicos asociados al desbalance energía-proteína del alimento, tal como se ha presentado en tilapias (Oreochromis niloticus) (Jiménez et al., 2008) y en la carpa común (Cyprinus carpio) (Erdal y Ercument, 2006). Por otro lado, se ha demostrado lipidosis hepática asociado a peroxidación de las grasas rancias (Caballero et al., 2002; Iregui, 2004), aunque en este caso se deberían observar lesiones necróticas en otros tejidos.

Las lesiones macroscópicas de hígado sugerentes de lipidosis hepática (coloración pálida, amarilla y de consistencia friable) se registró en el 39.1\% (9/23) de peces considerados como sanos, en tanto que mediante la observación de lesiones histológicas compatibles con lipidosis hepática se llegó a registrar en el $91.3 \%(21 / 23)$ de esta población. Esta diferencia se debe a que la observación macroscópica es más subjetiva, orienta pero no define; asimismo, los casos de lipidosis hepática leves (grados 1 y 2) sólo podrían detectarse de manera precisa mediante un examen histológico.

La presencia de truchas sanas con una frecuencia alta de lipidosis hepática en los grados 3 y 4 (30.4\%) cada uno, sugiere que este tipo de lesión no sería causante de signos clínicos en las truchas, lo que demostraría la capacidad que tiene la especie de soportar altos grados de severidad de lipidosis hepática. Esto ha sido demostrado en la tilapia, que soporta una alta acumulación de grasa en los hepatocitos, siendo un proceso normal en esta especie y no considerándose un proceso patológico (Iregui, 2004). La alta frecuencia de animales enfermos sin lesiones microscópicas (grado 0) compatibles con lipidosis hepática $(42.8 \% ; 15 / 35)$ indica que estas truchas estaban enfermas por causas diferentes a lipidosis hepática, pudiendo ser asociadas a causas infecciosas, como el hongo sistémico (Ictiofonus spp) y bacterias bacilares Gram (-) que fueron observadas en el parénquima hepático.

La alta frecuencia de trastornos infecciosos $(58.6 \%$; 34/58) asociado a ictiofonosis se debe a que esta enfermedad causa una infección sistémica caracterizada por una severa reacción inflamatoria crónica, de tipo granulomatosa. Esta enfermedad ha sido reportada en las piscigranjas del valle del Mantaro (Mateo, 1996), zona del presente estudio.

En los trastornos inflamatorios asociado a granulomas se encontraron bacterias Gram positivas y Gram negativas de forma bacilar, predominando las bacterias acidófilas. Entre los posibles agentes bacterianos que causan granulomas en el hígado como en otros tejidos en peces se encuentran Flavobacterium spp, Renibacterium spp y Aeromona spp (Kinkelin et al., 1995; Noga, 1996). Sin embargo, estos agentes tienen órganos blancos diferentes al tejido hepático.

\section{Conclusiones}

- La frecuencia total de lipidosis hepática en la trucha arcoíris (Oncorhynchus mykiss) en una piscigranja de la Sierra Central del Perú fue de 70.6\% (41/58).

- La elevada frecuencia de lipidosis hepática en grado 3 y 4 (escala de 0 a 4 ) en peces sanos sugiere la capacidad de la especie de soportar altos niveles de lipidosis hepática sin mostrar signos clínicos.

- La lesión macroscópica más común en el hígado fue la coloración pálida $(41.3 \%)$.

\section{Literatura CitTada}

1. Bernet D, Schmidt H, Meier W, Burkhardt-Holm P, Wahli T. 1999. Histopathology in fish: proposal for a protocol to assess aquatic pollution. J Fish Dis $22: 25-34$. 
2. Blanco C. 1995. La trucha, La cría industrial. $2^{\circ}$ ed. España: Ed Mundi Prensa Libros. 503 p.

3. Caballero M, Obach A, Rosenlund G Montero D, Gisvold M, Izquierdo M. 2002. Impact of different dietary lipid sources on growth, lipid digestibility, tissue fatty acid composition and histology of rainbow trout, Oncorhynchus mykiss. Aquaculture 214:253-271.

4. Carlyle T, Duncan D. 1990. Patología veterinaria. Vol I. $2^{\circ}$ ed. Buenos Aires: Ed Hemisferio. 310 p.

5. Cowey C, Roberts R. 1983. Nutritional pathology of teleosts. In: Roberts R (ed). Fish Pathology. London: Balliere-Tindall. p 249-255.

6. Cubillos V, López I, Alberdi A. 2001. Aspectos lesionales microscópicos en hígados de salmonídeos, provenientes de centros de cultivo de la X a XII Región de Chile. Periodo 1988-1998. Arch Med Vet 33: 203-216.

7. Erdal Y, Ercument G. 2006. Effects of alternative dietary lipid sources (Soyacid oil and yellow grease) on growth and hepatic lipidosis of common carp (Cyprinus carpio) fingerling: A preliminary study. Turkish J Fish Aquatic Sci 6: 37-42.

8. Ferguson H. 1989. Systematic pathology of fish. Canada: Iowa State University Press. 134 p.

9. Ferguson H. 2006. Systemic pathology of fish. $2^{\text {nd }}$ ed. Canada: Ed Scotian. 278 p.

10. Gang J, Feng J, Qin Z. 2006. Studies on the fatty liver diseases of Sciaenops ocellatus caused by different ether extract levels in diets. Front Biol China 1:9-12.

11. Hibiya T. 1982. An atlas of fish histology normal and pathological features. Tokio, Japan: Ed Kodansha. 147 p.

12. Iregui C. 2004. Primer mapa epidemiológico de las lesiones y enfermedades de los peces en Colombia Bogotá. Bogotá: Facultad de Medicina Veterinaria y Zootecnia, Univ Nacional de Colombia. $50 \mathrm{p}$.
13. Jiménez S, Herrera E, Hernández E, Guarín M, Wills A, Iregui C. 2008. Efecto de dietas isoenergéticas con diferentes niveles de proteína sobre algunos parámetros productivos y sobre algunos órganos de alevinos de tilapia Oreachromis nilótica variedad chitralada. En: Memorias II Curso Seminario Internacional de Ictiopatología. Colombia.

14. Kinkelin P, Michel C, Ghittino P. 1995. Tratado de las enfermedades de los peces. España: Ed Acribia. 124 p.

15. López I, Cubillos M, Ernst S, Rosenfeld C. 2001. Frecuencia y distribución, según edad y estacionalidad de patologías hepáticas en salmónidos, provenientes de la X a XII Región de Chile. Periodo 1988-1998. Arch Med Vet 33: 193-202.

16. Mateo D. 1996. La ictiofonosis y su prevalencia en las principales piscigranjas del valle del Mantaro. Tesis de Magíster. Lima: Facultad de Oceanografía, Pesquería y Ciencias Alimentarias, Univ Nacional Federico Virrarreal. 68 p.

17. Noga E. 1996. Fish disease: diagnostic and treatment. Iowa, USA: Iowa State University Press. 367 p.

18. Ostrander G. 2000. The laboratory fish. San Diego, USA: Academic Press. 678 p.

19. Roald S. 1976. An outbreak of liver lipoid degeneration (LLD) in Atlantic salmon in a fish farm and attempts to cure disease. Nord Vet Med 28: 243-249.

20. Roald S, Armstrong D. 1981. Histochemical, fluorescent and electron microscopical appearance of hepatocellular ceroidosis in the Atlantic salmon Salmo Salar L. J Fish Dis 4: 1-14.

21. Roberts J, Shepherd J. 1980. Enfermedades de la trucha y del salmón. Zaragoza, España: Ed Acribia.187 p.

22. Roberts $R .1983$. Fish pathology. $2^{\text {nd }} e d$. London: Balliere-Tindall. 318 p.

23. Spiegel M. 1991. Estadística. $2^{\circ}$ ed. EEUU: McGraw-Hill.

24. Trigo F. 1998. Patología general veterinaria. $2^{\circ}$ ed. España: Interamericana McGraw-Hill. 242 p. 